\title{
Evidence for Competition and Coordination between Vocal and Manual Responses in Preschool Children ${ }^{1}$
}

\author{
Davin Birch \\ Unitersity of Michigar
}

\begin{abstract}
Vocal and manual responses are peripherally compatible and could conecivably oecur completely independently of each other. Alternatively, processes controlling these responses might interact centrally following principles of competition and/or coordination. In order to initiate the investigation of this relationship vocal and manual response limes to a light were obtained from $35 \mathrm{Ss}$ agcd $3 \frac{1}{2}$ to $6 \frac{1}{2}$ years under two conditions; first in separate testing and then when hoth responses were called for by the light. Comparisons among the measures indicate the two response systems are not independent and suggest the hypothesis that processes of coordination occur with ehildren 4 yrars or older that do not oceur with younger children.
\end{abstract}

Making vocal responses (e.g., saying words) is compatible with making manual responses (e.g., moving the hand). This means that the two responses can take place at the same time of course but it also suggests the possibility that the system controlling the one response mode is independent of the system controlling the other. At least this possibility cannot be ruled out on the basis of peripheral characteristics of the two responses.

On the other hand, peripheral compatibility of the two responses does not insure that the systems controlling the responses are not interrelated at another level. For example, the two systems might utilize the same components in processes employed in stimulus reception or mediation or response production, giving rise to incompatibilities at a secondary level. Such incompatibilities might be accommodated in two distinctly different ways. Competition for the shared components could exist with the resulting conflict resolved in all or none fashion by one or the other system gaining the use of the required component at a particular moment in time. Under this arrangement, the two systems would function largely

'This research was carried out under the Program-Project on the Development of Language Functions in the Center for Human Growth and Development and was supported by NICH HD 01368-02. Robert. Wozniak and Linda Townes collected the data and assisted with the analyses. Mr. Wozniak and Adrienne Tentler made important contributions to the interpretation of the data through numerous discussions with the author. 
independently of each other but would meet in competition at one or more selected points in the processes governing the response productions.

The shared components could operate in a more complicated fashion, however, by providing for coordination between the two systems. For example, it might be that when, simultaneously, there are demands for a rocal response and a manual response, some pattern of organization is imposed on the production of the two responses. This could take the form of the one response consistently preceding the other in time or of the two taking place together or of a merging of the two original responses into a new response unit.

In the experiment to be reported, the type of relationship that obtains between a vocal and a manual response when both responses are called for by the same signal is explored. Specific hypotheses of independence, competition, and coordination are investigated, unguided by any preconreived opinions as to what these relationships might be. The data are gathered by having $S$ s respond over three different blocks of trials to the same light signal with either a vocal response alone, a motor response alone or both the vocal and motor responses. By comparing the latencies of the two responses obtained under the separate conditions with those obtained under the combined condition, the hypothesis that the vocal and manual response systems proceed independently of each other can be evaluated. The hypothesis of competition between the two response systems suggests examining the distributions of differences in latencies for vocal and manual responding under combined conditions to see if there is an unusual lack of entries in the interval around zero. Such a finding would be symptomatic of a difficully in performing the two responses simultaneously. The same distributions of differences provide the opportunity to look for evidence that the two response systems are coordinated. For example, an unusually large number of vocal-manual latency differences near zero in the combined condition would point to a process of synchronizing operative when both responses are called for by the same signal. Several specific hypotheses of independence, competition, and coordination are evaluated in this fashion.

There is a special reason for studying the relationship between a vocal and a manual response rather than, say, two peripherally compatible motor responses. By using one response from the verbal system and one not from that system, information can be gained about whether the verbal system performs a special dominating or controlling function with respect to the motor system. This is an important facet of the investigation of the verbal control of nonverbal behavior, a topic of some considerable theoretical and empirical interest recently (Luria, 1961; Bijou \& Baer, 1966; Birch, 1966; Miller, Shelton \& Flavell, 1970). 
There are also special ruasons for begiming the study of this problem with preschool fhildren. The derelopment of verbal control experially but of cognitive and notor control generally proceeds rapidly in the preschool vears, and clifforent ages in this range often reveal important differences in capacities and functions. Such might be the case for the relationship between vocal and manual responses.

Response requirements were kept simple in the experiment by using an adaptation of simple-reaction time methods." Initially, $S$ responded to the onset of a light by calling out the color (bluc) in one set of trials and by touching a target with his hand in another set of trials. Following this, a third set of trials was given in which 5 was instrueted to make both the manual and vocal responses to the onset of the light. The first two scts of trials provide basc-line reaction times for the two responses when only one or the other is called for, and the last set of trials gives the reaction times when both responses are called for. Evidence concerning the independence, competition, and coordination of the vocal and manual responses was sought in comparisons between the reaction times for the separate and combined conditions.

\section{METHOD}

\section{Subjects}

The Ss were 45 children attending an Ann Arbor nursery school supported by the United Fund and designed to serve mainly working mothers." The total sample consisted of 24 males and 21 females and included 30 white and 15 black children with an age range from $31 / 2$ to $61 \%$ years. The $S_{s}$ were assigned to experimental conditions so as to balance roughly these three factors across groups. Loss of data, primarily due to apparatus failure, reduced the usable sample size to 35 , composed of 18 males and 17 females.

\section{Apparatus}

The Ss were seated facing a horizontal response panel and an upright panel on which the colored light stimulus was presented. The latter is 9 in. high by 11 in. wide with a back-lit circular piece of frosted glass, $11 / 2$ in. in diameter, mounted at approximately eye level. The response panel, $13 \mathrm{in}$. wide by $20 \mathrm{in}$. long includes a start button $1 \mathrm{in}$. in diameter and a target plate $2 \frac{1}{2}$ by $3 \frac{1}{4} \mathrm{in}$. located directly in front of $S$. The distance between the button and the plate is adjustable and was set at 5 in. for all $S$ s.

"See Hohle (1967) for a review of the research on reaction times with ehildren.

${ }^{3}$ Special thanks are due Mrs. Elizabeth McHale, director of the Perry Nursery School, for her kind cooperation. 
One $E$, hidden from $S$ by a curtain, controlled the onset of the light and specified the response required to turn off the light by a set of silent switches. Three response requirements were set during the experiment. In one, called manual, the light went off when $S$ moved his hand from the start button and touched the target plate and in a sccond, called vocal, the light went off when $S$ called out the color of the light. In the third, called combined, both responses, touching the target plate and calling out the color of the light, were required but the two responses could be made in either order and with any latencies.

Three elucks, accurate to milliseconds were used to obtain latencies of responses to the onset of the light. One clock measured the time for $S$ to lift his finger from the start button following onset of the light (start time), a second the time taken to touch the target plate (target time), and a third clock recorded the latency of the vocal response (voice time). Contact switches were used to stop the clocks for the manual responses and a microphone, which $S$ wore, and a voice operated relay, located in another room, were used in connection with the rocal response.

\section{Procedure}

The experiment was conducted mornings in a trailer parked outside the school. The $E$ s played with the children in the nursery school for several days before beginning the experiment in order to get to know them and to gain their confidence. Total testing time was always less then $20 \mathrm{~min}$, and no rewards other than the company of the $E$ and the operation of the apparatus were provided. In general, younger children were tested before older children but beyond that no special order of testing was imposed.

Each $S$ responded under three conditions, Vocal, Manual, and Combined, corresponding to the three response requircments for turning out the light. For about one half of the $S s$ the order of conditions was vocalmanual-combined (designated Vocal-Manual) and for the remainder it was manual-vocal-combined (Manual-Vocal). In the combined condition where the children were told to make both responses, approximately one half of each of the Vocal-Manual and Manual-Vocal groups were instructed to "both say the name of the color and press the target" and one half to "both press the target and say the name of the color." This was done to assess the possibility that the order within the combined instruction would be a determinan of the order in which the child made the responses.

Nine trials were scheduled for each condition, three each at ready intervals of 2,4 , and 6 sec. These intervals were measured from the time $E$ said "ready" until the light came on, and were arranged in random order within each block of three trials. On a faulty trial, as occurred when the apparatus failed or the child missed the target plate, the same ready 
interval was repeated, either immediately or, in a few cases, after the other trials in the block had been completed.

Two $E s$ werc used. One recorded the clock readings, timed the ready intervals with a stop watch and turned on the stimulus. The child could sce this person but not the clocks or control devices. The other $E$ brought the child to the trailer, gave the instructions and observed the child as he performed.

The verbal explanations and demonstrations of the apparatus were similar but not identical for all $S$ s. The following instructions are typical:

Vocal. Now let's play a game. You watch right here and a light will come on. (Light goes on.) What color is it? O.K. Now you get to turn it off. Do you know how you can do it? O.K., then I'll show you. You put these on your head and, as soon as you see the light. you say the color-wateh. (Demonstrate.) Do you want io try it?

Manual. Now I'll show you a different way to play. (This was the introduction used for whichever condition was presented to the child second.) Put your finger on the red button and hold it down tight. $\Lambda_{s}$ soon as you sce the light, take your finger off the red button and touch the plate. Watch. Do you think you can do it?

Combined. Now we have one more way to play. This time you have to do two things to furn off the light: say the color and touch the plate (or vice versa). Romember to do them hoth. O.K. (No demonstration.)

\section{RFSULTS}

Data are complete for both the separate and combined conditions for $35 \mathrm{Ss}, 18$ males and 17 females. In the separate condition a block of nine trials of vocal responding preceded a block of nine trials of manual responding (Vocal-Manual order) for 10 males and 7 females, whereas the order of the two blocks was reversed (Manual-Vocal order) for 8 males and 10 females. These basic groups were divided further on the basis of instructions given in the combined condition when both vocal and manual responses were required on each trial.

Voice, target, start, and reach times (target time equals the sum of the start and reach times) are the measures of interest. The findings for these four measures from the separate condition will be summarized first, followed by the analyses from the combined condition. The latter are designed to assist in evaluating the hypotheses of independence, competition, and coordination concerning the vocal and manual systems.

\section{Separate Condition}

Figure 1 shows the mean voice, target, and start times for each of the three trial blocks and the two orders of testing under the separate condition. The Vocal-Manual subgroup has uniformly faster response times than the Manual-Vocal subgroup with this difference particularly pro- 
nounced for the target times. For both subgroups the target times are the longest and the voice and start times are approximately equal. The agreement between males and females for each of the response times justifies the pooling used in preparing Fig. 1.

For purposes of analyses of variance (three dimensional mixed designs with testing order, sex, and trial blocks as factors) all four cell frequencies were made equal to 10 by inserting three extra entries equal to the cell mean for the females in the Vocal-Manual subgroup and two extra entries for the males in the Manual-Vocal subgroup. Since the degrees of freedom employed in the analyses do not include these extra entries, no bias in the magnitude of the error terms results from this procedure. The ready intervals $(2,4$, or 6 sec) were ignored in detailed considerations of the data after preliminary analyses showed them not to be systematically related to any of the response times. Correlations between response times and age were assessed using Kendall's $\tau$, calculated separately for the two orders of testing using times averaged over all nine trials.

Briefly summarized, the results of the analyses for the four response times in the separate condition are as follows:

Voice. All simple effects of orders of testing, sex, trial blocks, and all interactions are nonsignificant in the analysis of variance. The correlation of voice time with age, calculated for the data pooled over males and

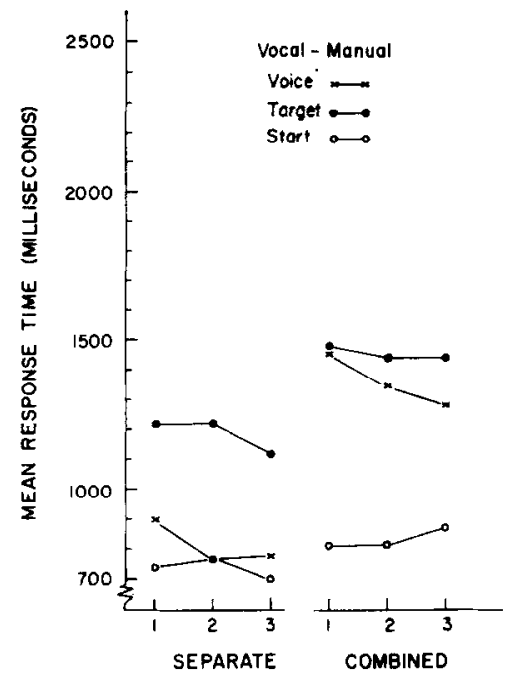

TRIAL BLOCKS

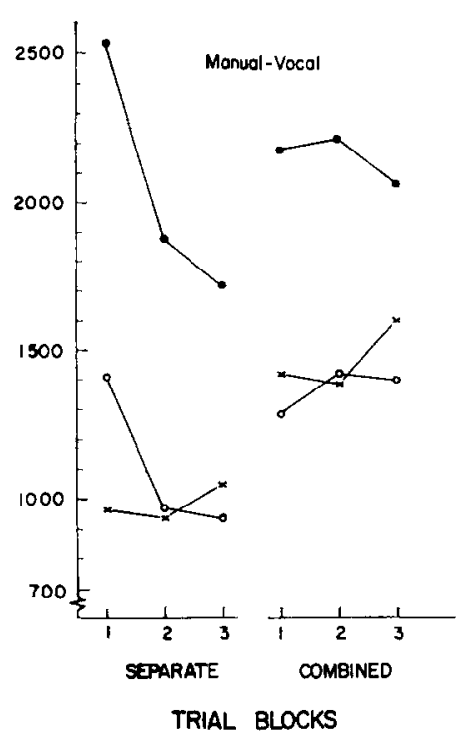

Fig. 1. Mean vocal, target, and start times for the Vocal-Manual and ManualVocal subgroups under the separate and combined conditions. 
females is $\tau=-\ldots .29$ (p< 2.10 ! when the vocal testing was prior to the uanual (Vocal-Manual orker), and $\tau=-.27(p)<.10)$ when it was subseguent to the manual. The rirection of the relationship, too weak to reach statistieal significance, is that the older sis atre faster than the younger st in their roeal responding.

Taryet. The overall separate condition mean target time of 1190 mser. for Sir given manual testing subseguent to rocal testing is significantly less that that of 2043 mire for siven manual testing first, $H^{2}(1,31)=$ 12.30, $p<.01$. In addition, as shown in Fig. 1. target times decrease across the three trial blocks ithe Trial Blocks effect yields $F(2,62)=$ a.28, $p<.001)$ and they do $\%$ to a greater extent when the manual testing occurs first the Trial Blocks $\times$ Testing Order interaction yields $F(2,62)-7.73, p<.0011$. All other effects in the analysis of variance, namely those related to the s'x of the Ss, fail to approach significance. The correlation of target response time with age is elearly nonsignificant for the Vocal-Manual testing order $(t \ldots-$ - 15$)$ bul approaches significanee when manual testing is carried out first $(\tau=-.32, p<.10)$, again with a trend in the direction of faster responding for the older children.

Stat. The results for the start response in the separate contition very largely repcat those for the target response. Overall, the mean start time is 737 mee when manual testing comes second and 1104 msec when it comes first, $F(1,31)=7.30, p<0.5$. The Trial Blocks main effect is significant, $F(2,62)=3.66, p<.05$, as is the Trial Blocks $\times$ Testing ( ) ror interaction, $F(2,62) \cdots 4.05, p<05$. In "ommon with the target moasure. the start times decline over the trial blocks and ro so much more dramatically when the manual testing oceurs first. No effects attributable to the sex of $S$ s approach significance. The correlation of start time with age is again negative and clearly nonsignificant when manual testing follows rocal testing $(\tau=-15)$ but approaches significance when manual testing comes first. $(\tau=-29, p<.10)$.

Reach. The overall mean response time for reaching is significantly faster for the Vocal-Manual testing order, 452 msec as against 939 msec for the Mimual-Vocal order, $F(1,31)=7.76, p<.01$. Over trial blocks the reaching times decrease signifieantly, $F(2,62)=3.57, p<.05$, but in contrast to the target and start measures the Trial Blocks $\times$ Testing croter interaction fails to reach significance, $F(2,62)=2.11, p>.10$. The correlations of reach time with age, $\tau=.01$ for the Vocal-Manual order and $z=-.15$ for the Manual-Tocal order, are clearly nonsignificant.

\section{Combined Condition}

As a rart of the intruetions for this condition ss were told cither to "say the color amd touch the plate" or to "touch the plate and say the 
color." In order to assess the effect of this difference in instructions each of the four response times in the combined condition was subjected to a preliminary analysis of variance. Because significant effects of testing order and trial blocks were found under the separate condition, these analyses were carried out on difference seores for each $S$, calculated by subtracting the mean response times for the last three trials of the separate condition from the oxerall mean response times for the nine trials: of the combined condition. The resulting two dimensional, instructions by sex of the $S$ s, factorial designs failed to prorluce any significant main effects or interactions. In fact, 8 of the $12 F$ ratios are less than imity. Accordingly, all further analyses were undertaken without regard to differences in the combined condition instructions.

Figure 1 includes the mean voice, target, and start times over the threc trial blocks of the combined condition for the two testing orders used in the separate condition. Most apparent in the figure are the consistently larger mean target and start times for the Manual-Vocal testing order. Less apparent hut also present is a lack of any particulas trend across trial blocks. Again, as in the separate condition, there is agreement in the patterns of response times for males and females.

A mixed design analysis of variane, with testing ordor in the separate condition, sex of the Ss, and trial blocks as factors was carried out on the data for each of the four response times. The ready intervals were not included in the analyses and entries equal to the cell means were inserted to bring all cell frequencies to 10 for the same rearons as applicd to the analyses of the separate condition data.

The findings are casily summarized. The only significant effects are attributable to the separate condition testing order, and then only for the target response, $F(1,31)=4.84, p<.05$, and for the start response $F(1,31)=6.62, p<.05$. These results bear out the differences apparent in Fig. 1.

No other effects, testing order, sex of the Ss or trial blocks, even approach significance in any of the analyses of variance. All eight correlations of response times with age (two $\tau^{\prime}$ 's werc computed for each response time, one for the Vocal-Manual order with $N=17$ and one for the Manual-Vocal order with $N=18$ ) are negative suggesting faster responding by the older $S$ s. But only one correlation, that for the target response when preceded by the Manual-Vocal testing order in the separate condition, is significant, $\tau=-.37, p<.05$.

\section{Evaluation of Hypotheses}

Next to be considered are the hypotheses of independence, competition, and coordination as applied to the verbal and notor systems in the 
present experiment. Figure 1 depicts graphically what happens to the mean voice, target, and start times as conditions change from separate: to combined responding. In carh case the times increase markedly and to approximately the same extent for the two subgroups.

The changes in mean respone time from the last three trials (Trial Block 3 ) of the separate condition to the overall average of the nine trials of the combined condition were evaluated by sign tests on the number of $S_{s}$ increasing their response times and by $t$ tests on the magnitudes of the increases. Results for the Vocal-Manual subgroup are: 16 of 17 Ss increase their voice times under combined conditions $(p<.001$ by the sign test) and the mean magnitude of increase is 577 msec $\left(t_{16}=6.63, p\right.$ $<.001) ; 14$ of $17 S_{\mathrm{s}}$ increase their target times $(p<.05)$ and the mean magnitude of inerease is $330 \mathrm{msec}\left(t_{16}=2.58, p<.05\right)$; and 13 of 17 Ss increase their start times $(p<.05)$ and the mean magnitude of increase is 125 msec $\left(t_{16}=1.68, p>.10\right)$.

The Manual-Vocal subgroup results are: 15 of 18 S have slower voice times in the combined $(p<.01$ by the sign test) with a mean increase of 419 msec $\left(t_{1 i}=5.11 . p<.001\right) ; 13$ of 18 Ss have slower target times $(p<.10)$ with a mean increase of 433 msec $\left(l_{17}=2.59, p<.05\right)$; and 14 of 18 Ss have slower start times $(p<.05)$ with a mean increase of 431 msec $\left(t_{1 i}=2.79, p<.05\right)$. Changes in the reach times are nonsignificant for both subgroups indicating that the increases in target times are primarily attributable to increases in the start times. As a consequence of these analyses the hypothesis that the peripherally compatible vocal and manual responses are unaffected by the combined responding condition and therefore arise from totally independent systems appears untenable.

A simple alternative to the independence hypothesis is that the vocal and manual systems are peripherally compatible but in competition centrally. It might be, for example, that only one or the other of the two actions, vocal or manual, can be initiated at a given moment in time. This could produce a queuing effect and an increase in response times. One place to look for symptoms of such competition is in the distribution of vocal minus start time differences $(V-S)$. An unusual scarcity of entries around zero in this distribution would give support to the competition hypothesis.

Table 1 represents the frequency distributions of $V-S$ for the VocalManual and Manual-Vocal subgroups using three intervals of approximately 100 msec centered around zero plus two additional intervals of considerably greater than $100 \mathrm{msec}$ used to accommodate the remaining entries at each end of the distributions. Separate groupings are made within the subgroups for the three trial blocks and for the ages of the Ss. Altogether there are only 15 entries in the $-49,49$-msec interval, 4 from 


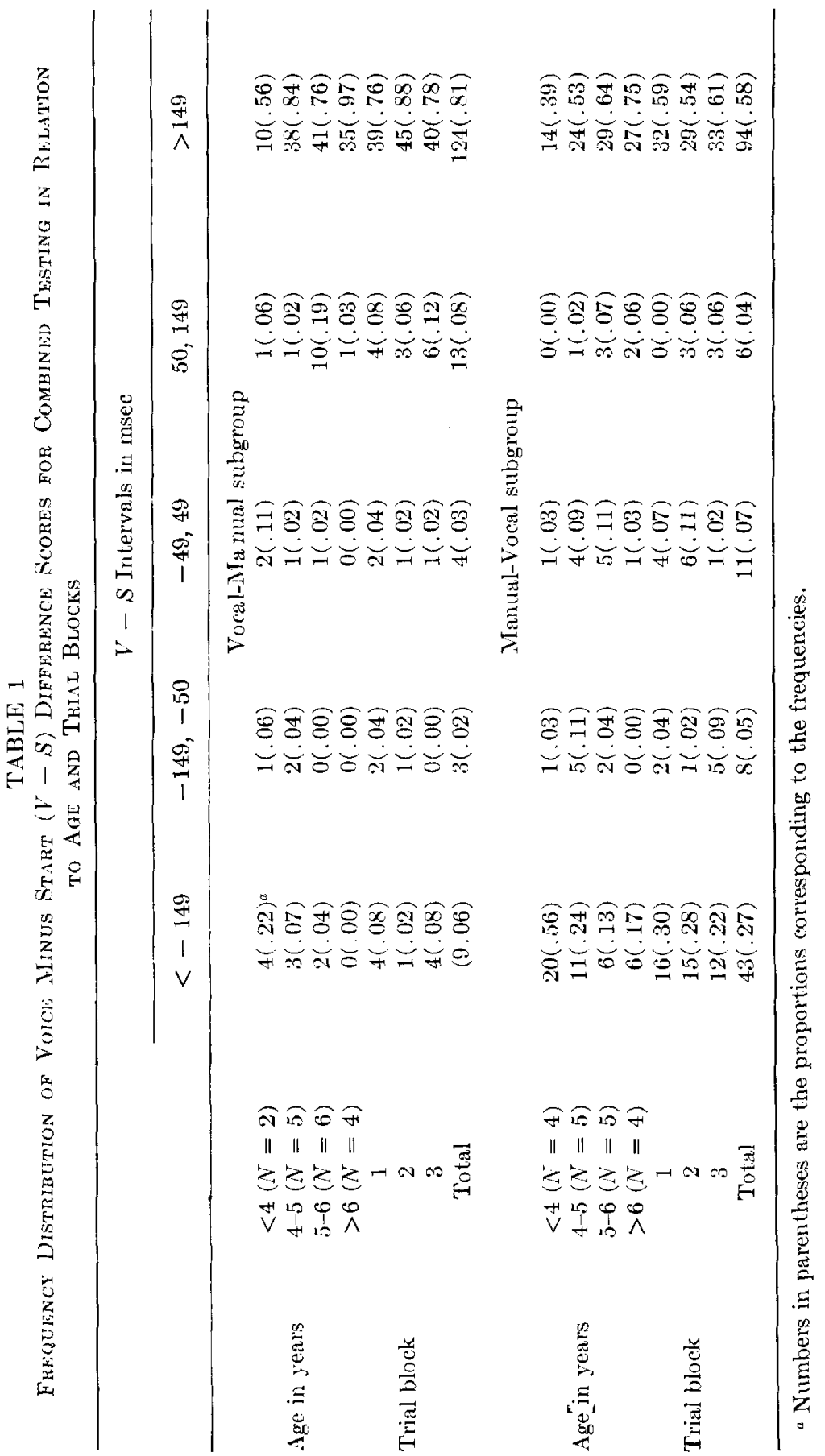


the Vocal-Manual sulgeroup and 11 from the Manual-Vocal. This apuears

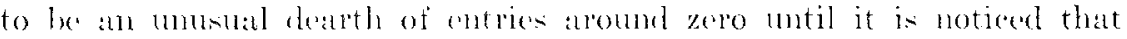
this intereal falls in the tails of the two distributions and that there atre relatively fow entries in any 100 -msere interval anywhere below 1 - $s$

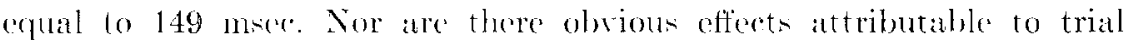
blocks or ages of the sis to be foum in Table 1. A proper evaluation of the competition hypothesis "amot he made from the data of this experiment, howerer, and must awat data that allow the opportumity for a depression around zero in the $F-S$ distribution to show.

The hypothesis that $S$ s synchronize their vocal and target responses. illustrating a type of coordination between the rerbal and motor systems. gains support from the combined conditions data. First, Fig. 1 shows the mean roice and target times for the Vocal-xanual subgroup to be much more nearly the same under the combined responding condition than under the separate combition. This is not true for the Manual-Voeal subgroup which applears to be frerforming the start and rocal responses at about the same time. Data conecrning moan response times do not bear dirently on the synchonizing hypothesis, howerer, since the means alone tell little about whether the vocal and target responses tend to ocanr together on catch trial.

A sccond and more satisfactory measure of synchronizing is obtained from the distribution of differences between the roice and target times $\left(T^{*}-T\right)$, particularly with respecet to the number of such differences in the neighborhoor of zero. Table 2 presents these data for the two subgroups with separate summaries for the trial hlocks and the ages of the Se wing the same intervals as employed in Table 1 for the $V-s$ differences:

Overall, the Vocal-Manual subgroup has $24 \%$ of its entries in the -49, 49-msere interval and the Manual-Vocal subgroup has $13 \%$. The mode of the distribution of $\mathrm{T}-T$ scores is in the $-49,49$ interval for the first subgroup and close to it for the second. Further examination of Table 2 shows that for the Vocal- Manual subgroup the degree of synchronizing, is a constant $24 \%$ across the three trial blocks but for the ManualVocal subgroup it incruses from 9 to 11 to $19 \%$. The $19 \%$ places the mode of the $r-T$ distribution in the -49 , 49-msec interval for the Manual-Vooal subsroup on the third trial block.

That okfre sis my sychronize more than younger ss is suggested by the positive, though nonsignificant, correlations between age and the numbers of entries in the $-49,49$-msere interval under combined responding. For the Vocal-Manual subgroup $\tau=.13, p>.50$ and for the Manual-Vocal subgroup) $\div .28, p<.10$. In addition, however, the data of Table 2 indicate that there may be a rather discrete onset of synchro- 


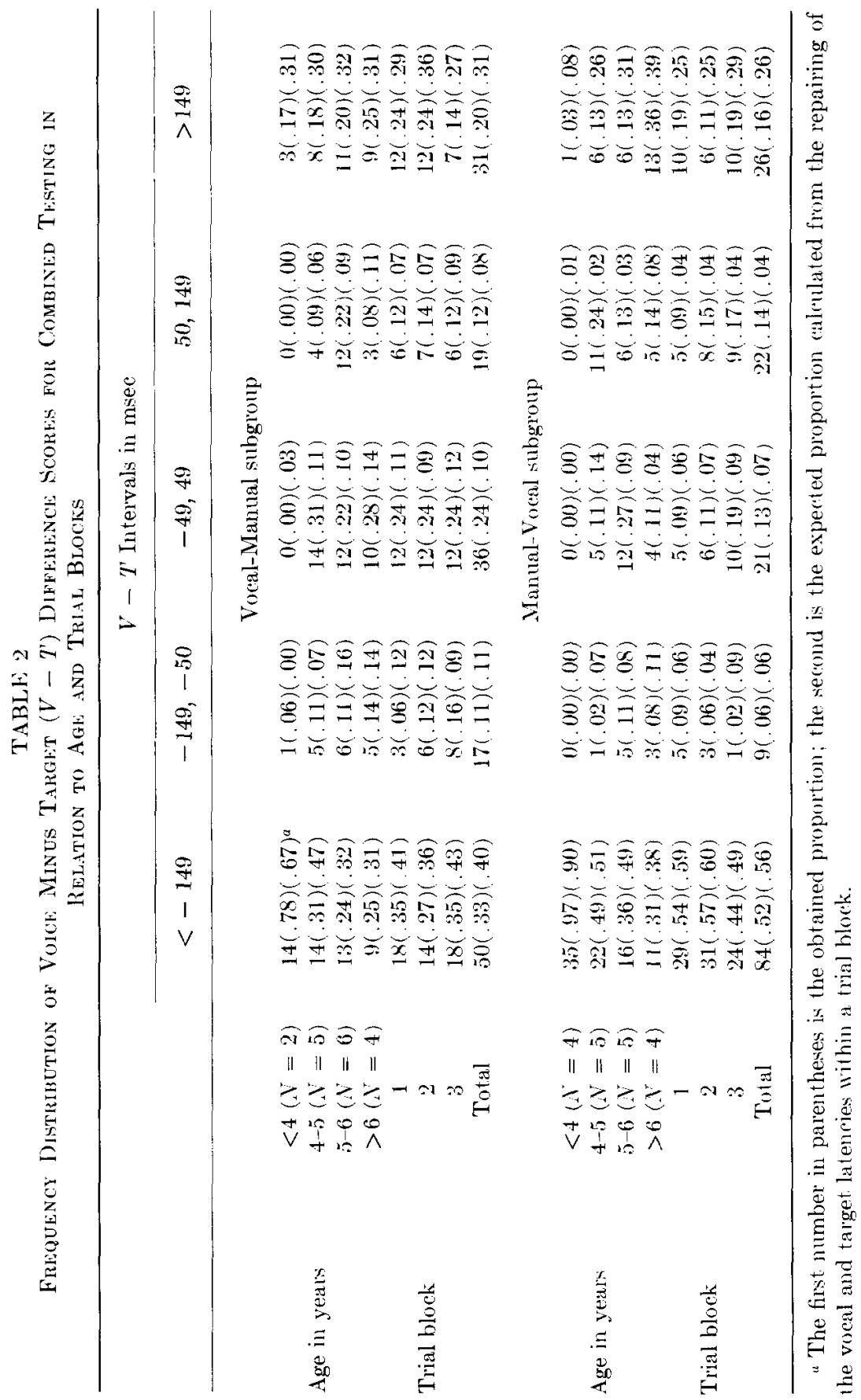




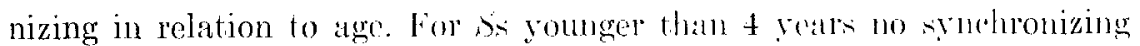
is to be found whereas for Se olker than 4 yotre the ocenrrener of synchronizing is quite uniform arross Ages 4,5 . and 6 yoars.

It is difficult to make a satisfactory statistical test of the hyrothesis that there is a significant degree of synchronizing occurring in the combined condition because it is not obvious what the obtained values should be compared against. One possibility, which perhaps provides a conservative test, is to generate the two additional $V-T$ distributions for each of the three trial blocks of the combined condition by repairing the three vocal and target times within each of the trial blocks. Thus, instead of pairing Trials 1, 2, and 3 for voice with Trials 1, 2, and 3 for target within Trial Block 1 as defined ly the combined condition, Trials 1, 2 , and 3 for voice can be paired with Trials 2, 3, and 1 and with Trials 3 , 1 , and 2 for target. This procolure generates a reference distribution of $V-T$ scores with the same mean as the obtained distribution.

The proportions resulting from the described repairings are included in Table 2 . In general, the calculated proportions are appreciably smaller than the obtained proportions for the $-49,49$ interval under all breakdowns in the table lending support to the synchronizing hypothesis. When evaluated by $\chi^{2}$ with the calculated proportions used to determine the expected frequencies, the discrepance betwen the observed and expected valucs is significant for both subgroups, $\chi^{2}(1)=31.12, p<.001$ for the Vocal-Manual Ss who have proportions of .24 and .10 and $\chi^{2}$ (1) $=8.95, p<.01$ for the Manual-Vocal $S_{s}$ who have proportions of .13 and .07. The expected frequencies are not large enough to test the discrepancies observable for age and trial blocks.

As might be anticipated some individuals synchronize more than others. For the Vocal-Manual subgroup $9 S_{s}$ (6 males, 3 females) have more entries in the $-49,49$ interval than would be expected on the basis of the repairing procedurc, $3 S s$ ( 1 male, 2 females) have the same number and $5 \mathrm{Ss}$ ( 3 males, 2 females) have fewer entries. The mean obtained number of entries in the $-49,49$ interval is 2.1 and the mean number calculated by the repairing procedure is 0.9 . A $t$ test for related measures shows the mean difference of 1.2 to be significant, $t(16)=3.00, p<.01$, giving statistical support to the synchronizing hypothesis. For the Manual-Vocal subgroup $5 \mathrm{Ss}$ ( 1 male, 4 females) have more entries in the -49 , 49 interval than expected, $10 S s$ (6 males, 4 females) have the same number and $3 \mathrm{Ss}$ (1 male, 2 females) have fewer. The mean obtained number of entries in the $-49,49$ interval is $\mathbf{1 . 2}$ and the mean calculated number is 0.7 . The mean difference, 0.5 , while in accord with the hypothesis of synchronizing by its direction, is not significant, $t(17)=1.25$, $p>.20$. 


\section{DISCUSSION}

The primary question behind the design of the experiment is:

Will the response times to a stimulus for two peripherally compatible responses, one vocal and the other manual, be different when obtained in separate testing than when obtained undcr conditions in which each presentation of the stimulus calls for the making of both responses?

The answer from the data is that the two conditions of testing do produce different response times.

Specifically, both mean voice and mean target times are significantly slower in the combined condition than in the separate condition (Fig. 1) with the increases in target times due to increases in start times rather than reach times.

While ruling out an hypothesis of strict independence between the vocal and manual response systems, the findings concerning the mean response times can be interpreted equally wcll by the hypotheses of competition and coordination. The competition hypothesis is quite consistent with the finding that under the combined condition the voice and start times are longer than under the separate condition. This result would be expected, for example, if neither response is initiated unless the child attends to it and if he is unable to attend to both responses at the same time. In this case calling out the color of the light and moving the hand would occur secquentially and with longer latencies, on the average, under the combined condition.

The competition hypothesis, however, has a major shortcoming and that is that it leaves unexplained the additional finding that under the combined condition calling out the color of the light and touching the target tend to occur together. This conclusion is based on the observation that the mode of the distribution of voice minus target times under the combined condition of responding sccms to be located in the 100-msec interval around zero (Table 2) and on the results of the statistical analyses with these data. Such synchronizing of the vocal and manual responses indicates that some type of coordinating or organizing is occurring. Under an hypothesis of coordinating one might well assume that the processes involved require time and thereby account for the overall delayed onset of the two responses under combined conditions.

Most damaging to the coordination hypothesis, perhaps, is that, when looked at in absolute terms, synchronizing is not found as often as it might be. (The figure is $18 \%$ overall for the combined condition.) On the other hand there is some indication that synchronizing is more likely to occur with older children, or at least may be quite unlikely to occur with children younger than 4 years (Table 2 ). This agrees with available 
information on the derelopment of language and agnitive rapacities in the child which points to rapid growth in these areas in the 3- to 4-year age rallge.

The major findinge from the experinent appear to be generalizable over the ready intervals used, the trials of the combined condition testing. the sex of the childen, and the phrasing of the instructions just prior to the combined condition testing. Fuch is not the case for the two orders of testing, imposed in the separate condition. Figure 1 shows the Vocal-Manual subgroup to be markedly faster in target responding than the Manual-Vocal subgroup. A difference between the two subgroups appears also in relation to the extent of synchronizing during the combined condition testing. When rocal testing takes place first and is followed by manual testing in the separite condition, $24 \%$ of the combined condition trials met the eriterion of synchronizing whereas for the reverse order of testing only $13 \%$ do so.

A reason for the different results from the two orders may be found in the nature of the two responses plus a general tendency in older children to coordinate compatible activities. The development of this reasoning, will be assisted by making two observations about the data of Fig. 1. First, the mean roice time for the Vocal-Manual subgroup is approximately 1200 msec faster than the mean target time for the Manual-Vocal subgroup). This comparison is between the first observations: on the two subgroups and therefore is not contaminated by practice effeets. It suggests that, initially, rocal responding is faster than manual responding in this experiment. The second observation is that practice on manual reponding has negligible effect on the subsequent mean roice time hut practice on vocal responding appears to lower the subsequent moan target time greatly.

This asymmetric result can be accounted for in tems of the coordinating hypothesis as follows: When rocal testing is given first, Ss learn to make a rocal response to the light. Then, assuming that the rocal response continues, albeit nonaudibly, during the subsecuent manual testing and that this rocal response synchronizes the target response, the latter could be expected to occur with approximately the same latency as the former as in the data. By the same argument, when manual testing comes first, si learn to make a manual response to the light. These target times are uninflucnced by the vocal response and tend to be quite long. During subsequent vocal testing the manual response is not made so the issue of srnehronizing docs not arise. Hence, the mean voice time for the Manual-Vocal subgroup could be expected to be comparable to that for the Vocal-Mamul subgroup as is the case. This set of assumptions accounts for the pattern of mean response times shown in Fig. 1. 
In addition, however, if synchronizing begins under the separate condition for the Vocal-Manual subgroup as assumed, it should continue into the combined condition and be present in Trial Block 1. In contrast, synchronization for the Manual-Vocal subgroup might be delayed and only gradually build during the combined condition testing. This additional expectation is also confirmed in the data. The occurrence of synchronizing under the combined conditions for the Vocal-Manual subgroup is constant at $24 \%$ for each of the three trial blocks but increases from $9 \%$ to $11 \%$ to $19 \%$ across trial blocks for the Manual-Vocal subgroup.

The speculations in the just preceding paragraphs include the assumption that the vocal response synchronizes the target response under certain conditions. Such would be the casc, for example, if the two occur together in time because the manual response is drawn to the vocal response by a process organized around the occurrence of the vocal response. This is an assumption that might properly be labelled "verbal control" in the sense that the vocal response is given a special or predominant role in the coordination of the rocal and manual responses. At the same time this assumption concerning the role of the verbal system in regulating motor behavior is weaker than one which proposes that the semantic rontent of the rocal response functions to direct motor hehavior.

The stronger assumption failed to receive support in the study by Miller, Shelton, and Flavell (1970). These investigators had children squeeze a ball in combination with vocalizations of "Squeeze" and "Don't Squeeze" according to the procedures of Luria (1961) and found little or no evidence that the vocalizations served a directive function over the manual responses. In reporting the details of their results, however, Miller et al. included the observation that the children in their study, aged $3-5$ years, tended to perform the vocal and manual responses at very nearly the same time (i.e., generally within $100-250$ msec of each other). This appears to be a finding of coordination in agreement with that of the present experiment. Unfortunately, the data of Miller et al. do not assist in determining whether the occurrence of the manual response is coordinated to the occurrence of the vocal response as proposed in the weaker form of the verbal control assumption.

\section{REFERENCES}

BrJoU, S. W., \& BaEr, D. M. Operant methods in child behavior and development. In W. K. Honig (Ed.), Operant beharior: areas of reveurch mul application. New York: Appleton-Century-Crofts. 1966.

Brach, D. Verbal control of nonverbal behavior. Journal of Experimental Child Psychology, 1966, 4, 266-275, 
Hohle, R. H. Component process latencies in reaction times of children and adults. In L. P. Lipsitt, and C. C. Spiker (Eds.), Advances in child development and behavior. New York: Academic Press, 1967.

LuRIA, A. R. The role of speech in the regulation of normal and abnormal behavior. Now York: Liveright, 1961.

Miller, S. A., Shelton, J., \& Flaveld, J. H. A best of Luria's hypolheses concerning the development of verbal self-regulation. Child Development, 1970, 41, 651-665. 